(Aus der Universitäts-Frauenklinik zu Halle. Director: Geheimrath Prof. Dr. J. Veit.)

\title{
Untersuchungen ïber den Gehalt des Blutplasmas an Gesammteiweiss, Fibrinogen und Reststickstoff bei Schwangeren.
}

Ein Beitrag zur Frage der Beziehungen zwischen Leukocytenzerfall und Entstehung fibrinbildender Substanzen und deren Bedeutung für die Schwangerschaftsniere und Eklampsie.

Von

\section{Erich Landsberg,}

cand. med.

Das Verhalten des Blutes in der Gravidität, und insbesondere die Beziehungen zwischen mütterlichem und fötalem Blute sind im letzten Jahrzehnt Gegenstand lebhaften Interesses geworden. Allerdings sind schon in früherer Zeit Blutuntersuchungen bei Schwangeren und Neugeborenen ausgeführt worden, aber abgesehen von dem Umstande, dass die verwendeten Methoden den neuzeitlichen Ansprüchen vielfach nicht zu entsprechen vermögen, beging man bei den Untersuchungen des Nabelschnurblutes den Fehler, die gefundenen Resultate zu vergleichen mit den für den Erwachsenen festgestellten Zahlen.

Soweit ich aus der Literatur ersehen kann, war J. Veit (1) der Erste, welcher in einer grösseren Versuchsreihe das fötale Blut direct mit dem möglichst gleichzeitig entnommenen mütterlichen Blute verglichen hat, eine Forderung, an der man trotz der oft nicht unbeträchtlichen Schwierigkeit der Ausführung unbedingt festhalten muss. Seiner Arbeit über den osmotischen Druck zwischen Mutter und Kind, in welcher er als Erster die Kryoskopie, die Lehre von der Gefrierpunktserniedrigung, der Erforschung der Stoffwechselvorgänge zwischen Mutter und Kind dienstbar machte, folgten bald eine grosse Anzahl anderer Autoren, welche das Blut von Schwangeren und Neugeborenen in morphologischer, biologischer, physikalischer und chemischer Hinsicht studirten. 
694 Landsberg, Untersuchungen über den Gehalt des Blutplasmas u.s.w.

Es würde zu weit führen, wollte ich all' der Forscher Erwähnung thun, welche auf diesem Gebiete gearbeitet haben. Wer sich über die einschlägige Literatur informiren will, findet eine sehr vollständige Zusammenstellung im Referat von Heymann (2).

Hat es also an Bemühungen nicht gefehlt, die einzelnen Fragen der Blutbeschaffenheit zu klären, so ist dieses Ziel doch nur zum Theil erreicht worden, indem die einzelnen Ergebnisse vielfach von einander abweichen. Ganz besonders gilt dies auch von den Eiweissbestimmungen, eine Thatsache, die wohl am besten aus einer Anführung der in den hauptsächlichsten Arbeiten gefundenen Mittelwerthe sich erkennen lässt.

\section{Frühere Untersuchungen über das Gesammteiweiss.}

Zangemeister (3): Schwangere 63,2 pM., Kreissende 66,8 pM., gesunde, nicht schwangere Frauen 81,5 pM., angegebenes Literaturmittel $84,5 \mathrm{pM}$.

Zangemeister und Meissl (4): Mntter 67,9 pM., Kind 51,6 pM. Lewinski (5): Schwangere 76,7 pM., gesunde Nichtschwangere $72,9 \mathrm{pM}$.

Scipiades und Farkas (6): Schwangere 75,0 pM., Kreissende $76,0 \mathrm{pM}$., Wöchnerinnen $73,5 \mathrm{pM}$.

Raineri (7) findet im Blute von Schwangeren die Eiweissmenge verringert und giebt an, dass im Fötalblut der Gehalt an Proteinen grösser sei als im mütterlichen Blute.

Bauereisen (8) nennt für den Eiweissgehalt des Nabelschnurblutes 55,0 pM., für denjenigen des Blutes aus dem retroplacentaren Hämatom 81,5 pM. als Durchschnittszahl, wobei jedoch gesagt werden muss, dass dieser Autor den gefundenen Stickstoffgehalt mit 6,37 ! multiplicirt.

Wie man sieht, handelt es sich hier nicht um kleine Zahlendifferenzen, welche durch individuelle Schwankungen oder durch kleine Unterschiede in der Versuchsausführung sich erklären liessen, sondern um directe Widersprüche in der Beantwortung der grundlegendsten Fragen.

Zangemeister, Meissl und Raineri finden das Bluteiweiss in der Schwangerschaft geringer, Lewinski, Scipiades und Farkas im Gegensatz dazu grösser als bei der normalen Frau. Im Fötalblut stellen Zangenmeister, Meissl und Bauereisen eine Verminderung, Raineri hingegen eine Vermehrung der Eiweissmenge im Vergleich zum mütterlichen Blute fest. 
Landsberg, Untersuchungen über den Gehalt des Blutplasmas u.s.w. 695

Eine genaue Nachprüfung dieser Ergebnisse an einer möglichst grossen Zahl von Fällen schien also sehr erwünseht.

In - nevester Zeit begnügt man sich aber nicht mehr mit der Feststellung der gesammten Eiweissmenge, sondern man ist auch bemüht, die einzelnen Eiweisskörper quantitativ von einander zu scheiden und ihr Verhältniss bei den einzelnen pathologischen $\mathrm{Zu}$ ständen zu erforschen.

Mit Recht weisen Langstein und Meyer (9) darauf hin, dass in dieser. Hinsicht nur die Untersuchung des Blutplasmas nutzbringend sein kann. Gerade der specifische Eiweisskörper des Blutplasmas, das Eibrinogen, aus welchem bekanntlich bei der Gerinnung das Fibrin sich bildet, scheint bei einzelnen Erkrankungen ein constantes Verhalten zu zeigen. Es kann nur mit Freude begrüsst werden, dass auch die Geburtshelfer und Gynäkologen ihr Interesse diesen für die normale wie pathologisehe Physiologie gleichwichtigen Fragen zugewendet haben.

\section{Frühere Fibrinogenbestimmungen.}

Die bisher im normalen menschlichen Blutplasma vorgenommenen Fibrinogenbestimmungen sind nur spärlich. Ich habe in der Literatur folgende Zahlenangaben gefunden:

$$
\begin{aligned}
& \left.\begin{array}{rrr} 
& \\
\text { Efeiffer (10): } & 0,3490 \mathrm{pCt} \\
0,4251 & \\
0,3661 & \\
0,3235 & n
\end{array}\right\} \text { Männer } \\
& \text { Lewinski (5): Männer Frauen } \\
& 0,36 \text { pCt. } \quad 0,27 \text { pCt. } \\
& 0,48 n 0,35 n
\end{aligned}
$$

Hieraus ergeben sich folgende Mittelwerthe des Fibrinogengehaltes:

$$
\text { Männer 0,384 pCt., Frauen 0,31 pCt. }
$$

Auf Veranlassung von Dienst, auf dessen Arbeiten ich später eingehen werde, hat Lewinski das Fibrinogen bei normalen Schwangeren und Eklamptischen bestimmt.

Frühere Untersuchungen hatten nämlich gezeigt, dass der Fibringehalt bei Schwangeren und besonders bei Eklamptischen grösser ist als in der Norm. Die diesbezüglichen Literaturangaben sind: 
696 Landsberg, Untersuchungen äber den Gehalt des Blutplasmas u.s.w.

Nasse (12): Fibrin bei Nichtschwangeren i. M. . . 2,36 pM.

" im 6.-8. Schwangerschaftsmonat 3,354 "

" im 9. Schwangerschaftsmonat . . 3,673"

"während des Kreissens . . . . 3,82 "

Kollmann (13): Fibrin bei Eklamptischen 9,0pM.

$\left.\begin{array}{l}7,5 \% \\ 6,7 \pi\end{array}\right\}$ i. M. $7,7 \mathrm{pM}$.

Dienst (14): Fibrin bei Schwangerschaftseklampsie. . 2,8 pM.

" "Eklampsie während der Geburt 3,87 "

" "Wochenbettseklampsie . . . 4,665"

Ich möchte nicht unterlassen, auf den beträchtlichen Unterschied hinzuweisen, welcher zwischen den Untersuchungsergebnissen von Kollmann und Dienst besteht. Die von dem erstgenannten Autor angegebenen Zahlen müssen wegen ihrer ausserordentlichen Höhe recht fraglich erscheinen. Nach Dienst zeichnet sich nur die Wochenbettseklampsie durch einen erheblich gesteigerten Fibringebalt aus.

Bei dem Vergleich der Zahlen für das Fibrin und das Fibrinogen muss man sich stets die Thatsache vor Augen halten, dass ganz allgemein weniger Eibrin entsteht, als dem vorhandenen Fibrinogen entspricht.

Lewinski (5) findet die folgenden Mittelzahlen für das Fibrinogen:

2 nicht schwangere Frauen. . 0,31 pCt. (s. o.)

4 normale Schwangere . . 0,45 "

3 Eklamptische. . . . . 0,53"

1 Eklamptische. . . . . 0,32, !

1 Urämische . . . . 0,26"

Der Forscher sagt von seinen Untersuchungen bei den Eklamptischen selbst Folgendes:

„Das Fibrinogen zeigt in diesen 3 Fällen eine weitere, aber sebr geringe Zunahme gegenüber der Schwangerschaft." Im vierten Falle war sogar eine deutliche Abnahme des Fibrinogens zu constatiren. Lewinski giebt auch selbst zu, dass seine Untersuchungen nicht im Stande sind, die Fibrinogenfrage zu entscheiden, indem die Zahl ron 2 Nichtschwangeren und 4 Schwangeren nicht ausreichend ist, um sichere Schlüsse daraus ziehen zu können. Als besonders beachtenswerthes Moment kommt noch hinzu, dass Lewinski bei den Eklamptischen die Blutentnahme "auf der Höhe 
Landsberg, Untersuchungen über den Gehalt des Blutplasmas u.s.w. 697 der Erscheinungen", d. h. also während oder kurz nach der Geburtsarbeit vorgenommen hat, wohingegen or dies bei normalen Schwangeren nicht gethan. Von vornherein musste es für sehr wahrscheinlich gelten, dass bereits bei Kreissenden sich höhere Fibrinogenwerthe als bei Schwangeren im 8 . und 9. Monat finden würden, zumal dies für das Fibrin bereits von Nasse festgestellt worden ist.

Ich habe mich bemüht, durch zahlreiche Untersuchungen die hier vorhandenen Lücken auszufüllen.

\section{Methoden zur Fibrinogendarstellung.}

Die erste Vorbedingung zur quantitativen Fibrogenbestimmung ist das Verhindern der Blutgerinnung. Dieses lässt sich auf verschiedenen Wegen erreichen.

Noch heute erfreut sich in dieser Hinsicht die von Arthus und Pagès (15) angegebene Verwendung von kalkfällenden Mitteln einer grossen Beliebtheit, ein Verfahren, welches auf der Thatsache beruht, dass die Anwesenheit von Kalksalzen eine nothwendige Bedingung für das Zustandekommen der Gerinnung ist. Arthus und Pagès empfahlen, das Blut aufzufangen in einer Lösung von Kaliumoxalat oder Fluornatrium und zwar so, dass der Gehalt des Blutes an Kaliumoxalat $0,1 \mathrm{pCt}$., an Fluornatrium $0.3 \mathrm{pCt}$. beträgt.

Diese Zahlen haben späterhin eine geringe Abänderung erfahren, indem Hammersten (16) $0,25-0,3 \mathrm{pCt}$. Kialumoxalat, Reye (17) $0,56-0,6$ pCt. Fluornatrium als optimalen Salzgehalt empfehlen.

Die Darstellung des Fibrinogens aus dem Blutplasma ist mit recht erheblichen Schwierigkeiten verknüpft. Zur Klasse der Globuline gehörig theilt es mit diesen die chemischen Eigenthümlichkeiten und kann nur durch Anwendung einer ganz bestimmten Concentration der Fällungsmittel von diesen gesondert erhalten werden.

Bereits Denis (18), welcher als Erster eine Isolirung des Fibrinogens versuchte, benutzte zu diesem Zweeke das Kochsalz. Indessen fallen bei der von ihm verwendeten Ganzsättigung auch andere Globulineiweisskörper des Blutes aus, und erst Hammersten hat die folgende brauchbare Methode angegeben:

Das durch Kaliumoxalat ungerinnbar gemachte Blut wird kräftig centrifugirt. Das abgeheberte klare Plasma bleibt ca. 24 Stunden bei einer Temperatur von ca. 0 Grad stehen, und es scheidet sich dann meist ein geringfügiger Niederschlag ab, welcher entfernt wird. 
698 Landsberg, Untersuchungen über den Gehalt des Blutplasmas u.s.w.

Die Flüssigkeit wird nun mit $1 / 2$ Vol. gesättigter kalkfreier Kochsalzlösung versetzt und der entstehende, sehr geringfügige Niederschlag abfiltrirt. Dem Filtrat wird dann $1 \frac{1}{2}-2$ Vol. ges. Kochsalzlösung zugesetzt, der entstehende Niederschlag in 8proc. Kochsalzlösung gelöst, durch Zusatz von 1 Vol. ges. Kochsalzlösung das Fibrinogen wiederum gefällt und dies dreimal wiederholt. Der zuletzt entstehende Niederschlag wird in Wasser gelöst, die Lösung durch Diffusion gegen 0,003 proc. Natronlauge salzfrei gemacht und schliesslich das Fibrinogen durch Alkohol abgeschieden, bis zur Gewichtsconstanz getrocknet und gewogen.

Durch Heubner (19) und Huiscamp (2) hat diese Methode einige Ergänzungen erfahren, die ich an dieser Stelle aber nicht näher schildern zu sollen glaube.

Ein zweites, neueres Verfahren zur Fibrinogenbestimmung stammt ron Reye (17).

Bereits Kander (21) zeigte, dass man durch fractionirte Fällung mit Ammoniumsulfat eine gute quantitative Trennung der Globuline von den Albuminen erzielen kann. Reye führt den Nachweis, dass dieses Salz auch zur Fibrinogenfällung äusserst geeignet ist, indem seine Untersuchungen ergaben, dass Fibrinogen bei $2,5-2,8 \mathrm{ccm}$ (obere Grenze) gesättigter Ammoniumsulfatiösung pro $10 \mathrm{ccm}$ Flüssigkeit bei $2 \mathrm{ccm}$ Plasma, Serum-Globulin hingegen erst bei $2,9 \mathrm{ccm}$ (untere Grenze) ausfällt. Er empfiehlt folgendermaassen vorzugehen:

Zu $12 \mathrm{cem}$ Fluornatriumplasma $(0,56-0,6$ pCt. Salzgehalt) fügt man $30 \mathrm{ccm}$ Aqua dest. und $16 \mathrm{ccm}$ ges. Ammoniumsulfatlösung. Der schnell sich absetzende Niederschlag wird nach ca. 4 Stunden auf einem aschefreien Filter gesammelt und mit entsprechend verdünnter Ammoniumsulfatlösung (42 T'eile Aqu. dest. und 16 Teile ges. Lösung), so lange gewaschen, bis sich das Filtrat als völlig eiweissfrei erweist. Alsdann lässt man den Niederschlag lufttrocken werden, coagulirt ihn ca. 12 Stunden bei 85 Grad, wäscht ihn mit heissem Wasser, Alkohol und Aether sehr grändlich aus, trocknet und wägt ihn.

Beide Methoden können nicht als völlig genau gelten. Durch Halbsättigıng mit Kochsalz erhält man zwar einen sehr reinen, aber, besonders bei Verarbeitung kleinerer Blutmengen, quantitativ zu geringen Fibrinogenniederschlag, während andererseits bei dem Verfahren von Reye, welches im Allgemeinen zur vergleichenden 
Landsberg, Untersuchungen über den Gehalt des Blutplasmas u.s.w. 699 quantitativen Bestimmung geeigneter zu sein scheint, die Reinheit des Niederschlages von manchen Seiten angezweifelt wird (22).

Was die technische Ausführung anbelangt, so ist an und für sich die Reye'sche Methode weniger zeitraubend als die von Hammersten. Letatere kann man aber dadurch sehr vereinfachen, dass man das Eibrinogen aus seiner Lösung durch die Stickstoff bestimmung nach Kjeldahl (23) berechnet, ein Verfahren, welches bei sorgfältiger Ausführung durchaus brauchbare Resultate liefert. Bei der Fällung mit Ammoniumsulfat lässt sich die Wägung nicht umgehen, weil es sich hier um ein stickstoffhaltiges Salz handelt.

Lewinski (5) hat bei seinen Untersuchungen die Nethode von Hammersten in sehr vereinfachter Form angewendet. Er versetzt 10-15 ccm Oxalatplasma mit $15-25 \mathrm{cem}$ ges. Kochsalzlösung, sammelt den Niederschlag nach einigen Stunden auf einem aschefreien Filter, wäscht ihn mit einer Kochsalzlösung von 15 Theilen ges. Lösung mit 25 Theilen Wasser aus bis das Filtrat völlig eiweissfrei, und bestimmt seinen Stickstoffgehalt nach Kjeldahl.

Anfangs hatte ich gegen dieses Verfahren, welches durch seine grosse Einfachheit imponiren muss, mancherlei Bedenken. Lewinski setzt $1 \frac{1}{2}-1 \%$ Vol. ges. Kochsalzlösung hinzu und spricht den ganzen entstehenden Niederschlag als Fibrinogen an, während von Hammersten zur endgültigen Abscheidung dieses Eiweisskörpers nur 1 Vol. ges. Lösung benutzt wird. Nun sagt dieser Autor aber selbst von dem Zusatz von $1 \frac{1}{2}-2$ Vol. ges. Salylösung zu 1 Vol. Oxalatplasma, dass dieses "ohne Gefahr für cine Verunreinigung mit Paraglobulin geschehen kann." Das Paraglobulin steht bekanntlich dem Fibrinogen am nächsten und eine Verunreinigung mit diesem Eiweisskörper ist daher am ehesten zu befürchten. Bedenkt man fernerhin, dass, wie bereits oben erwähnt, bei der Ausführung der Hammersten'schen Methode ein Quantitätsverlust sich kaum vermeiden lässt, so wird man das Vorgehen von Lewinski für durchaus haltbar erachten müssen.

Trotzdem würde ich der Ammoniumsulfatfällung den Vorzug gegeben haben, wenn ich nicht die von mir mit den von Lewinski gefundenen Werthen direct hätte vergleichen wollen. Hierzu war eine möglichst weitgehende Uebereinstimmung in der Versuchsausführung ein unbedingtes Erforderniss. 


\section{Eigene Versuche,}

Meine Untersuchungen erstrecken sich auf 18 Schwangere vom 6. bis 10. Monat mit drei Wiederholungen; 10 Kreissende, 6 Neugeborene, 8 nichtschwangere in Bezug auf die Blutbeschaffenheit normale Frauen. Ein Fall von Dedem und Albuminurie in der Gravidität.

Ausserordentlich bedauere jeh, dass es mir nicht möglich war, mehr pathologiscbe Fälle, namentlich auch Eklampsiekranke zu untersuchen, wie ich es beabsichtigt hatte. Unter diesen Umständen ist es mir besonders angenehm, dass ich in der Methodik fast genau mit Lewinsk i übereinstimme.

\section{Versucbsausführung.}

50 bis $60 \mathrm{ccm}$ Blut, der Vena mediana bezw. der schnell unterbundenen Nabelschnur entnommen, wurden in einem Kolben von ca. $65 \mathrm{ccm}$ aufgefangen, der vorher mit $3 \mathrm{ccm}$ einer 5 proc. Kaliumoxalatlösung beschickt worden war. Hierzu möchte ich bemerken, dass ich in den von mir angestellten Vorversuchen einen Vorzug der Fluornatriumlösung vor der Kaliumoxalatlösung, wie er von Reye angegeben wird, nie habe constatiren können. Das so ungerinnbar gemachte Blut wurde nun sofort zunächst eine halbe Stunde und dann nochmals drei Viertelstunden kräftig centrifugirt. Hierdurch liess sich am besten jeder Uebertritt von Stoffen aus den Formelementen in die Blutflüssigkeit, insbesondere auch jegliche Hämolyse vermeiden, was sonst wenigstens beim kindlichen Blute häufig nicht der Fall ist. Das klare Plasma wurde 12-24 Stunden bei Eiskühlung aufbewahrt und alsdann filtrirt. Auf diese Weise habe ich durchschnittlich von der Mutter $35 \mathrm{ccm}$, vom Kinde $25 \mathrm{ccm}$ eines völlig klaren, sehr haltbaren Oxalatplasmas gewonnen.

In diesem bestimmte ich das Fibrinogen; das Gesammteiweiss; den Gesammtstickstoff.

\section{Fibrinogenbestimmung.}

$10 \mathrm{ccm}$ Plasma wurde in einer Porcellanschale mit $15 \mathrm{~cm}$ chemisch reiner, gesättigter Kochsalzlösung versetzt, der entstehende Niederschlag nach ca. 6 Stunden auf einem aschefreien Filter gesammelt und mit halbgesättigter Kochsalzlösung bis zur völligen Eiweissfreiheit des Filtrates gewaschen.

\section{Gesammteiweissbestimmung (23b).}

In einer geräumigen Porcellanschale wurde $2-3 \mathrm{ccm}$ Plasma in ca. 50-75 ccm kochende Aq. dest. eingetragen und der kochenden Flüssigkeit ganz vorsichtig unter ständigem Umrühren tropfenweise 2 proc. Essigsäure zugesetzt so lange bis das Eiweiss in grösseren Flocken austiel und die darüber befindliche Flüssigkeit völlig klar erschien. Das Filtrat darf keine Spur von Eiweissreaktion geben.

Dieses Verfahren verlangt zur genauen Ausführung einiger Uebung. Hat man sich dieselbe angeeignet, so kann man mit ihm sehr genaue Resultate erzielen.

Sowohl das Filter mit dem Fibrinogen wie das mit dem Gesammteiweiss wurde verascht und der Stickstoff nach Kjeldahl bestimmt. Die Verascbung des Fibrinogenniederschlages muss anfangs sehr vor- 
Landsberg, Untersuchungen über den Gehalt des Blutplasmas u.s.w. 701

sichtig über kleiner Flamme geschehen, weil es in Folge des beträchtlichen Salzgehaltes leicht zum Ueberspritzen kommt.

Aus der Stickstoffmenge erhält man das Gesammteiweiss durch MuItiplication mit 6,25, das Fibrinogen durch Multiplication mit 100/16,66 $=6$, indem der Stickstoffgehalt des Fibrinogens 16,66 pCt. beträgt.

In 2-3 cem Plasma wurde schliesslich noch der Gesammtstickstoff bestimmt und durch Subtraction des Eiweissstickstoffs vom Gesammtstickstoff der Reststickstoff erhalten. Bei der Mutter wurde jeder Versuch doppelt ausgeführt, wäbrend beim Kinde wegen des geringeren Materials dieses meist nur für die Fibrinogenbestimmung möglich war.

Versuchsresultate.

Tabelle I. Schwangere.

\begin{tabular}{|c|c|c|c|c|c|c|c|c|c|}
\hline 离 & 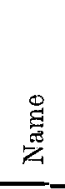 & 量 & $\begin{array}{c}\text { Zeit } \\
\text { der } \\
\text { Gravidität }\end{array}$ & $\begin{array}{l}\text { Eiweiss-N } \\
\text { in } 100 \mathrm{cem} \\
\text { Oxalat- } \\
\text { plasma } \\
\mathrm{g}\end{array}$ & $\begin{array}{c}\text { Ge- } \\
\text { sammt- } \\
\text { Eiweiss } \\
\text { in } \\
\text { pCt.g }\end{array}$ & $\begin{array}{c}\text { Fibrinogen- } \\
\text { Nin } 100 \mathrm{cem} \\
\text { Oxalat- } \\
\text { plasma } \\
\mathrm{g}\end{array}$ & $\begin{array}{l}\text { Fibri- } \\
\text { nogen } \\
\text { in } \\
\text { pCt.g }\end{array}$ & $\begin{array}{l}\text { Gesammt- } \\
\text { stickstoff } \\
\text { in } 100 \mathrm{ccm} \\
\text { Oxalat- } \\
\text { plasma g }\end{array}$ & $\begin{array}{c}\text { Rest- } \\
\text { stickstoft } \\
\text { in } 100 \mathrm{ccm} \\
\text { Oxalat- } \\
\text { plasma g. }\end{array}$ \\
\hline 1 & N. & II. & X. Monat. & 0,966 & 6,04 & 0,08 & 0,48 & 1,001 & 0,035 \\
\hline 2 & E. & I. & X. Monat. & 1,130 & 7,06 & 0,07 & 0,42 & 1,152 & 0,022 \\
\hline 3 & M. & I. & X. Monat. & 1,060 & 6,63 & 0,09 & 0,54 & 1,099 & 0.039 \\
\hline 4 & W. & I. & X. Monat. & 1,085 & 6,78 & 0,087 & 0,52 & 1,110 & 0,025 \\
\hline$\tilde{5}$ & H. & III. & VIII. Monat. & 0,994 & 6,21 & 0,065 & 0,39 & 1,105 & 0,021 \\
\hline 6 al & T & 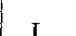 & / VIII. Monat. & 0,959 & 6,00 & 0,056 & 0.33 & 0,979 & 0,020 \\
\hline $6 \mathrm{~b}\}$ & L. & 1 . & RX. Monat. & 1,012 & 6,32 & 0,077 & 0,46 & 1,040 & 0,028 \\
\hline 7 & D. & I. & VIII. Monat. & 1,015 & 6,34 & 0,078 & 0,47 & 1,039 & 0,024 \\
\hline 8 & J. & I. & IX. Monat. & 1,029 & 6,43 & 0,088 & 0,53 & 1,049 & 0,020 \\
\hline 9 & L. & II. & VIIL. Monat. & 1,050 & 6,56 & 0,061 & 0,37 & 1,069 & 0,019 \\
\hline 10 & K. & $\mathrm{I}$. & IX. Monat. & 1,022 & 6,39 & 0,077 & 0.46 & 1,044 & 0,022 \\
\hline 11 al & $\mathrm{R}$ & T & (VI. Monat. & 1,060 & 6,63 & 0,051 & 0,306 & 1,085 & 0,025 \\
\hline $11 \mathrm{~b}\}$ & B. & 1. & KIII. Monat. & 1,072 & 6,70 & 0,064 & 0,38 & 1,091 & 0.019 \\
\hline 12 & B. & I. & X. Monat. & 1,085 & 6,78 & 0,083 & 0,50 & 1,108 & 0,023 \\
\hline 13 a) & & & SVII. Monat. & 1,120 & 7,00 & 0,074 & 0,44 & 1,151 & 0,031 \\
\hline $13 \mathrm{bJ}$ & U. & 11. & IIX. Monat. & 1,106 & 6,90 & 0,070 & 0,42 & 1,135 & 0.029 \\
\hline 14 & W. & 11. & IX. Monat. & 1,085 & 6,78 & 0,063 & 0.38 & 1,110 & 0,025 \\
\hline 15 & H. & I. & X. Monat. & 0,994 & 6,21 & 0,077 & 0,46 & 1,017 & 0,023 \\
\hline 16 & Seh. & I. & VII. Moment. & 1,036 & 6,48 & 0,056 & 0,336 & 1.056 & 0,020 \\
\hline 17 & H. & I. & VIIS. Monat. & 0,980 & 6,13 & 0,067 & 0,40 & 0,999 & 0.019 \\
\hline 18 & B. & I. & IX. Monat. & 1,130 & 7,06 & 0,058 & 0,35 & 1,153 & 0,023 \\
\hline
\end{tabular}

In $100 \mathrm{ccm}$ Oxalatplasma sind demnach enthalten bei Schwangeren im

VI. bis X. Monat.

im Max.: Fibrinogen $0,54 \mathrm{~g}$, Gesammteiweiss $7,06 \mathrm{~g}$

Reststickstoff: $0,039 \mathrm{~g}$

im Min.: Fibrinogen $0,306 \mathrm{~g}$, Gesammteiweiss $6,00 \mathrm{~g}$ Reststickstoff: $0,019 \mathrm{~g}$

im Mittel: Fibrinogen 0,426 g, Gesammteiweiss 6,54 g

Reststickstoff: $0,024 \mathrm{~g}$

In $100 \mathrm{eem}$ Oxalatplasma beträgt der Fibrinogengehalt:

bei Schwangeren im VI. u. VII. Monat:

im Max. $0,44 \mathrm{~g}$, im Min. $0,306 \mathrm{~g}$

im Mittel $0,36 \mathrm{~g}$;

bei Schwangeren im VIII. bis X. Monat:

im Max. 0,54 g, im Min. 0,33 g

im Mittel: $0,437 \mathrm{~g}$ 
702 Landsberg, Untersuchungen über den Gehalt des Blutplasmas u.s. w.

Tabelle II. Kreissende.

\begin{tabular}{|c|c|c|c|c|c|c|c|c|c|}
\hline$\underset{\stackrel{\Phi}{\Xi}}{\stackrel{\Xi}{\Xi}}$ & 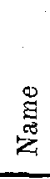 & 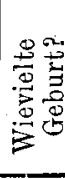 & $\begin{array}{c}\text { Zeit } \\
\text { der } \\
\text { Blutentnahme }\end{array}$ & $\begin{array}{c}\text { Eiweiss-N } \\
\text { in } 100 \mathrm{ccm} \\
\text { Oxalat- } \\
\text { plasma } \\
\mathrm{g}\end{array}$ & $\begin{array}{c}\text { Ge- } \\
\text { sammt- } \\
\text { Eiweiss } \\
\text { in } \\
\text { pCt.g }\end{array}$ & $\begin{array}{c}\text { Fibrinogen- } \\
N \text { in } 100 \text { ccm } \\
\text { Oxalat- } \\
\text { plasma } \\
\mathrm{g}\end{array}$ & $\begin{array}{l}\text { Fibri- } \\
\text { nogen } \\
\text { in } \\
\text { pCt.g }\end{array}$ & $\begin{array}{c}\text { Gesammt- } \\
\text { stickstoff } \\
\text { in } 100 \mathrm{ccm} \\
\text { Oxalat- } \\
\text { plasma g }\end{array}$ & $\begin{array}{c}\text { Rest- } \\
\text { stickstoff } \\
\text { in } 100 \mathrm{ecm} \\
\text { Oxalat- } \\
\text { plasma g }\end{array}$ \\
\hline 1 & W. & II. & 3/4 Std. ante part: & 1,134 & 7,09 & 0,076 & 0,46 & 1,166 & 0,032 \\
\hline 2 & St. & II. & $1 / 2$ Std. post part. & 1,029 & 6,43 & 0,0896 & 0,54 & 1,050 & 0.021 \\
\hline 3 & L. & I. & $1_{1 / 2}$ Std. post part. & 1,015 & 6,34 & 0,088 & 0,53 & 1,039 & 0,024 \\
\hline 4 & H. & I: & 1/4 Std. post part. & 0,959 & 6,00 & 0,086 & 0,52 & 0,980 & 0,021 \\
\hline 5 & St. & I. & 4 Std ante part. & 1,043 & 6,52 & 0,089 & 0,53 & 1,070 & 0,027 \\
\hline 6 & B. & I. & gleichzeit. m. part. & 1,085 & 6,78 & 0,094 & 0,56 & 1,105 & 0,020 \\
\hline 7 & $\mathrm{~K}$. & II. & gleichzeit. m. part. & 1,008 & 6,30 & 0,076 & 0,46 & 1,022 & 0,016 \\
\hline 8 & M. & I. & 1/2 std. post part. & 1,064 & 6,65 & 0,085 & 0,51 & 1,085 & 0,021 \\
\hline 9 & $H$. & I. & I Std ante part. & 1,155 & 7,20 & 0,080 & 0,48 & 1,177 & 0,022 \\
\hline 10 & P. & I. & 1/2 Std. ante part. & 1,019 & 6,37 & 0,070 & 0,42 & 1,049 & 0,030 \\
\hline
\end{tabular}

* Anm.: Partus bedeutet hier das Durchtreten des Kopfes.

Tabelle III. Nengeborene.

\begin{tabular}{|c|c|c|c|c|c|c|c|}
\hline 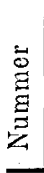 & $\begin{array}{l}\stackrel{0}{\Xi} \\
\text { 艺 }\end{array}$ & $\begin{array}{c}\text { Eiweiss-N } \\
\text { in } 100 \mathrm{~cm} \\
\text { Oxalatplasma } \\
\mathrm{g}\end{array}$ & $\begin{array}{c}\text { Fibrinogen-N } \\
\text { in } 100 \mathrm{ccm} \\
\text { Oxalatplasma } \\
\mathrm{g}\end{array}$ & $\begin{array}{c}\text { Gesammt-N } \\
\text { in } 100 \mathrm{cem} \\
\text { Oxalatplasma } \\
\mathrm{g}\end{array}$ & $\begin{array}{l}\text { Gesammt- } \\
\text { eiweiss in } \\
\text { pCt.-g }\end{array}$ & $\begin{array}{c}\text { Fibrinogen } \\
\text { in } \\
\text { pCt.-g }\end{array}$ & $\begin{array}{c}\text { Reststickstoff } \\
\text { in } 100 \text { ccm } \\
\text { Oxalatplasma } \\
\mathrm{g}\end{array}$ \\
\hline 1 & W. & 0,770 & 0,031 & 0,789 & 4,81 & 0,186 & 0.019 \\
\hline 2 & $\mathrm{~L}$ & 0,763 & 0,038 & $\ldots$ & 4,79 & 0,23 & - \\
\hline 3 & B. & 0,770 & 0,037 & 0.795 & 4,81 & 0,22 & 0,025 \\
\hline 4 & $\mathrm{~K}$. & 0,810 & 0,043 & 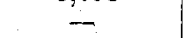 & 5.06 & 0,26 & - \\
\hline 5 & M. & 0,861 & 0,035 & 0,876 & 5,38 & 0,21 & 0,015 \\
\hline 6 & P. & 0,875 & 0,050 & - & 5,46 & 0,30 & - \\
\hline
\end{tabular}

In 100 ecrn Oxalatplasma sind demnach enthalten bei Kreissenden:

im Max.: Fibrinogen $0,56 \mathrm{~g}$, Gesammteiweiss $7,20 \mathrm{~g}$

Reststickstoff $0,032 \mathrm{~g}$

im Min.: Fibrinogen 0,42 g, Gesammteiweiss 6,00 g

Reststickstoff $0,016 \mathrm{~g}$

im Mittel: Fibrinogen 0,50 g, Gesammteiweiss $6,57 \mathrm{~g}$

Reststickstoff $0,0234 \mathrm{~g}$.

In 100 com Oxalatplasma sind demnach enthalten bei Neugeborenen:

im Max.: Fibrinogen $0,30 \mathrm{~g}$, Gesammteiweiss $5,46 \mathrm{~g}$

Reststickstoff $0,024 \mathrm{~g}$

in Mia.: Fibrinogen $0,19 \mathrm{~g}$, Gesammteiweiss $4,79 \mathrm{~g}$

Reststickstoff $0,015 \mathrm{~g}$

in Mittel: Fibrinogen 0,24 g, Gesammteiweiss $5,05 \mathrm{~g}$

Reststickstoff $0,020 \mathrm{~g}$. 
Landsberg, Untersuchungen über den Gehalt des Blutplasmas u.s.w. 703

Tabelle IV. Nichtschwangere Frauen.

\begin{tabular}{|c|c|c|c|c|c|c|c|c|}
\hline 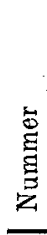 & 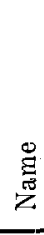 & Krankheit & 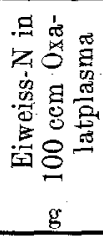 & 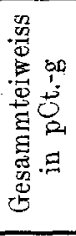 & 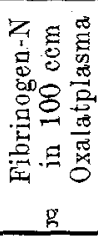 & 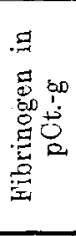 & 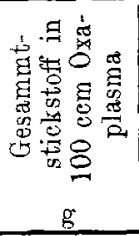 & 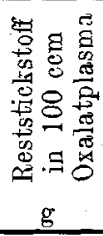 \\
\hline 1 & M. & Retroflexio: keine Blutungen & 1,141 & 7,13 & 0,056 & 0,33 & 1,155 & 0,014 \\
\hline 2 & $\mathrm{Z}$. & Descensus vag: do. & 1,160 & 7,25 & 0,067 & 0,40 & 1,181 & 0,021 \\
\hline 3 & H. & Retroflex.mob.: & 1,085 & 6,78 & 0,057 & 0,34 & 1,110 & 0,025 \\
\hline 4 & J. & do. & 1,106 & 6,91 & 0,060 & 0,36 & 1,125 & 0,019 \\
\hline 5 & D. & do. & 1,092 & 6,83 & 0,0714 & 0,43 & 1,120 & 0,020 \\
\hline 6 & K. & Retroflexio: & 1,113 & 6,96 & 0,067 & 0,40 & 1,135 & 0,022 \\
\hline 7 & $\mathrm{H}$. & Prolapsus vag. & & 7,00 & 0,0742 & 0,445 & 1,144 & 0,024 \\
\hline 8 & E. & Hysterie & 1,155 & 7,22 & 0,513 & 0,31 & 1,175 & 0,020 \\
\hline
\end{tabular}

In $100 \mathrm{ccm}$ Oxalatplasma sind demnach enthalten bei nichtschwangeren Frauen, deren Blutzusammensetzung als normal angesehen werden kann:

im Max.: Fibrinogen $0,445 \mathrm{~g}$, Gesammteiweiss $7,22 \mathrm{~g}$

im Min.: Fibrinogen $0,31 \mathrm{~g}$, Gesammteiweiss $6,78 \mathrm{~g}$ Reststickstoff $0,025 \mathrm{~g}$

im Mittel: Fibrinogen $0,377 \mathrm{~g}$, Gesammteiweiss $7,01 \mathrm{~g}$

Restatickstoff $0,014 \mathrm{~g}$

Reststickstoff $0,021 \mathrm{~g}$.

\section{Ein Fall von 0edemen und Albuminurie in der Sehwangerschaft.}

E's handelte sich hier $1 \mathrm{~m}$ eine Schwangere, deren Urin 1,5 pM. Eiweiss enthielt. Im Sediment waren zahlreiche Gylinder nachweisbar. Starke Oedeme.

Die Blutentnahme geschah 10 Tage vor der Entbindung. Es ergaben sich folgende Werthe:

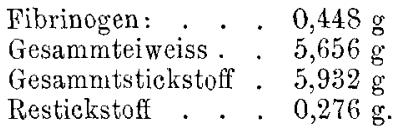

Eine vergleichende Betrachtung zeigt, dass meine Ergebnisse gut übereinstimmen einerseits mit den von Zangemeister und Meissl (3 und 4) für das Gesammteiweiss und den Reststickstoff, andererseits von Lewinski (5) für das Fibrinogen gefundenen Werthen.

Nach meinen Untersuchungen ist der Gesammteiweissgehalt im Mittel bei Schwangeren bezw. Kreissenden geringer als bei Nichtschwangeren: 6,54:6,57:7,01. Bei Neugeborenen geringer als bei der Mutter: $5,03: 6,57$.

Fibrinogengehalt im Mittel bei Schwangeren etwas grösser als bei Nichtschwangeren: $0,426,(6 .-10$. Monat): $0,437,(8 .-10$. Monat): 0,377. Bei Kreissenden noch höher als bei Schwangeren: $0,507: 0,437$. Bei Neugeborenen in 0 pCt. auf die Gesammteiweissmenge berechnet, nicht nur geringer als bei Kreissenden, sondern auch niedriger als bei den Nichtschwangeren: 4,7 pCt., 7,6 pCt., 5,36 pCt. Der Reststickstoff zeigt keine bemerkenswerthen Verschiebungen.

In dem einen pathologischen Fall ist einerseits die geringe Ge- 
704 Landsberg, Untersuchungen über den Gehalt des Blutplasmas u.s.w.

sammteiweissmenge - 5,65 pCt. - andererseits der sehr hohe Reststickstoffgehalt - $0,276 \mathrm{pCt}$. - bemerkenswerth. Leider bat es Lewinski unterlassen bei den Eklamptischen den Reststickstoff zu bestimmen. Mit grösster Wahrscheinlichkeit lässt sich auch bei diesen eine starke Steigerung vermuthen. Der Fibrinogengehalt $-0,448 \mathrm{pCt}$ ist keineswegs gegenüber den Werthen der normalen Sehwangerschaft erhöht.

Neben der letzten Thatsache möchte jch besonders den Punkt hervorheben, dass schon bei normalen Kreissenden der Fibrinogengehalt gegenüber der Schwangerschaft ziemlich beträchtlich gesteigert ist.

Wie schon gesagt, findet Lewinski bei Eklamptischen im Mittel 0,53 pCt. Fibrinogen. Im Vergleich mit der für die letzten Schwangerschaftsmonate festgestellten Mittelzahl - 0,45 pCt. glaubt er eine „sehr geringe Zunahme" nicht ausschliessen zu können. In Wirklichkeit liegen aber die Verhältnisse anders. Stellt man, wie es zur richtigen Beurtheilung der Frage erforderlich ist, die Werthe der normalen Kreissenden und der Eklamptischen gegenüber, so sieht man, dass von einer Fibrinogensteigerung bei letzteren nicht die Rede sein kann.

Noch besser als an den Durchschnittszahlen lässt sich dieses an den Einzelresultaten zeigen. Bei der Mehrzahl der Kreissenden, ja selbst bei 2 Schwangeren finden sich Werthe über $0,5 \mathrm{pCt}$. im Maximum 0,56 pCt. bezw. 0,54 pCt. - obwohl es sich um durchaus normale Fälle handelt.

Diese Feststellungen erscheinen mir gerade jetzt interessant.

Seitdem besonders durch die Untersuchungen von Schmorl (24) und Lubarsch (25) festgestellt worden ist, dass für das pathologische Bild der Eklampsie eine ausgedehrte Thrombose charakteristisch ist, wird von einigen Seiten die Ansicht vertreten, dass für diese Erkrankung das ätjologische Moment mehr oder weniger in einem starken Anwachsen gerinnungsbildender Substanzen zu suchen sei.

Dienst (26) hat nun in einer kürzlich erschienenen Arbeit auf Grund seiner nach der Arneth'schen Methode ausgeführten Untersuchungen über das Verhalten der weissen Blutkörperchen bei der Schwangerschaftsniere und Eklampsie eine neue eigenartige Theorie aufgestellt, die ich im Folgenden kurz zusammenfassen möchte.

Bei den genannten pathologischen Zuständen stellt Dienst eine Hyperleukocytose fest, welche sich nicht nur in quantitativer, 
sondern, was er für die Hauptsache hält, auch in qualitativer Hinsicht von dem Leukocytenbilde bei der normalen Schwangerschaft unterscheidet. Während nämlich im letzteren Falle die Zahl der ein- und zweikernigen polynucleären Leukocyten diejenige der vielkernigen bedeutend übertrifft, findet sich bei der Schwangerschaftsniere und besonders bei der Eklampsie das umgekehrte Verhältniss, d. h. also ein starkes Prävaliren der vielkernigen polynucleären Leukocyten.

Von der Annahme ausgehend, dass es gerade diese Blutkörperchen sind, welche bei ihrem Zerfall diejenigen Substanzen liefern, die das Fibrin entstehen lassen, d. h. also einerseits das Fibrinogen, andererseits das Fibrinferment, in Sonderheit Thrombokinase, glaubt er, dass durch einen starken Zerfall dieser so zahlreich vorhandenen Leukocyten die fibrinbildenden Substanzen in reichlicher Menge entstehen und ihre schädigende Wirkung auf den mütterlichen Organismus ausüben.

Bei der Schwangerschaftsniere geht dieser Vorgang nur allmählich vor sich, und die entstehende Thrombokinase kann durch die im Blutplasma normaler Weise vorhandenen gerinnungshemmenden Körper unschädlich gemacht werden. Nicht so das Fibrinogen. Angeblich soll das Fibrinogen die Fähigkeit besitzen, das Endothel der Capillaren zu schädigen und so zur Entstehung von Oedemen und, falls auch die glomerulären Capillaren der Nieren ergriffen werden, zur Albuminurie Veranlassung geben können.

Bei der Eklampsie hingegen kommt es zu einer ganz plötzlichen Ueberschwemmung der oberen Körperhälfte mit den fibrinerzeugenden Substanzen, und es bilden sich zahlreiche Thrombosen, namentlich auch in den Hirngefässen, welche die eklamptiscben Anfälle hervorrufen.

Zur Begründung dieser Anschauung zieht Dienst den Umstand heran, dass es sich bei Eklamptischen meistentheils um Erstgebärende oder um eine mehrfache Schwangerschaft handelt, d. h. also um Frauen, bei denen in Folge straffer Bauchdecken oder abnormer Füllung des Uterus eine sehr starke Blutstauung in den unteren Extremitäten eintritt. Gerade hier sollen sich nun die vielkernigen polynucleären Leukocyten ansammeln und bald in Folge der schlechten Circulationsverhältnisse degeneriren. Beim Eintritt der Wehen gelangen diese degenerirten Leukocyten in grosser Zahl in den allgemeinen Kreislauf, und die freiwerdenden beträchtlichen 
706 Landsberg, Untersuchungen über den Gehalt des Blutplasmas u.s.w.

Mengen von Fibrinogen und Thrombokinase können zahlreiche Thrombosen hervorrufen.

Dienst spricht schliesslich die Vermuthung aus, dass alle Frauen, die an Schwangerschaftniere oder Eklampsie erkranken, auch schon vor diesen Zuständen eine Hyperleukocytose quantitativer nicht aber qualitativer Natur gehabt haben.

Es kann keinem Zweifel unterliegen, dass die angeführte Theorie nur durch wenig Thatsächliches gestützt ist. Wenn Dienst sagt, Lewinski hat gefunden, dass bei Eklamptischen der Fibrinogengehalt "gegenüber der Norm um ein Bedeutendes vermehrt ist", so entspricht dies nicht den Thatsachen. Nach meinen Untersuchungen lässt sich, wie schon hervorgehoben, sowohl für die Eklampsie wie auch mit Wahrscheinlichkeit für die Schwangerschaftsniere ein Anwachsen des Fibrinogens ausschliessen. Auch wird man gewisse Bedenken gegen so weitgehende Schlussfolgerungen aus der Leukocytendifferenzirung nach Arneth kaum unterdrücken können, denn dieser Punkt kann heute noch keineswegs als völlig geklärt gelten.

Vor Allem erscheint es wichtig, die Frage eingehend zu untersuchen, in wieweit nach den geltenden physiologischen Anschauungen Leukocytenzerfall und Fibrinvermehrung zusammenhängen.

Hierzu muss ich ausführlicher auf den Process der Blutgerinnung eingehen.

Seit den Untersuchungen von Alex Schmidt (28) und Hammersten (29) ist es eine feststehende Thatsache, dass das Fibrin durch eine fermentative Umwandlung des Fibrinogens entsteht. Ueber den Charakter des Fibrinfermentes ist eine völlige Einigung trotz der sehr zahlreichen Arbeiten noch nicht erzielt worden.

Die im Folgenden ausgeführten, sich an die Anschauungen von Morawitz (30), Feld und Spiro (31) anschliessenden Hauptpunkte der Gerinnungslehre geniessen jetzt die weitgehendste Anerkennung und sie liegen auch den Ausführungen von Dienst zu Grunde.

Das Fibrin entsteht durch Einwirkung des activen Fibrinfermentes, d. h. Thrombin, auf das Fibrinogen. Das Thrombin bildet sich aus dem im Blutplasma präexistirenden Thrombogen und der nicht präformirten Thrombokinase unter dem Einfluss von Kalksalzen. Bei dem Gerinnungsvorgang wird nicht alles gebildete Thrombin verbraucht, vielmehr ist es auch im Serum vorhanden, 
Landsberg, Untersuchungen über den Gehalt des Blutplasmas u.s.w. 707 allerdings zum überwiegenden Theil in einer unwirksamen Form, Metathrombin; dieses Metathrombin kann durch Zusatz von Alkalien und Säuren activirt werden.

An dieser Stelle interessirt vor Allem die Herkunft des Fibrinogens und der Thrombokinase.

\section{Herkunft des Fibrinogens.}

Ursprünglich glaubte man, dass das Fibrinogen erst extravasculär beim Zerfalle der geformten Blutelemente entstehe.

Diese Anschaung ist bereits durch Joh. Müller (32) widerlegt worden, welcher bewies, dass "der Faserstoff in flüssiger Form" sich im Blute vorfindet.

Die verschiedensten Organe sind seither mit der Fibrinogenresp. Fibrinbildung in Zusammenhang gebracht worden, so von Lehmann, Brown-Séquard, Cl. Bernard u. A. m. die Haut und besonders der Darm, von Dastre (33) die Lunge, welche nach diesem Eorscher auch gleichzeitig fibrinzerstörend wirken soll.

A. Schmidt (28) behauptete, dass aus dem in den Zellen vorhandenen Cytoglobin zunächst das Paraglobin und dann das Fibrinogen entsteht. Diese ganz unbewiesene Hypothese hat kaum einen Anhänger gefunden, und es ist unzweifelhaft, dass das Fibrinogen direct aus zelligen Elementen hervorgeht.

Die Anschauung, dass der Darm die Ursprungsstätte des Fibrinogens sei, hat, obwohl lediglich auf der unsicheren Behauptung beruhend, dass das Mesenterialvenenblut reicher an diesem Eiweisskörper sei als das arterielle Blut, eine allgemeine Annahme gefunden.

In neuerer Zeit ist diese Ansicht besonders von Mathew (34) verfochten worden, welcher die Einwirkung der Herausnahme des Darmes bei der Katze studirte. Er kam zu dem Resultat, dass nach gänzlicher Defibrination eine Wiederbildung des Fibrinogens nicht stattfindet, wenn der Darm vorher entfernt worden ist. Unter Betonung des nach seiner Meinung stets vorhandenen Parallelismus zwischen Lenkocytenzahl and Fibrinmenge stellt er die Behauptung auf, dass durch den Zerfall der Leukocyten namentlich des Darmes das Fibrinogen entsteht.

Auch Corin und Ansiaux, welche die Beobachtung machten, dass nach Phosphorvergiftung das Biut ungerinnbar wird, sprechen die Läsionen des Darmes als die Ursache für das Ausbleiben der 
708 I andsberg, Untersuchungen über den Gehalt des Blutplasmas u.s.w.

Gerinnung an. In der Leber sehen sie den Zerstörungsort des Fibrinogens.

Diese Meinung stammt bereits von Lehmann (36), welcher angiebt, dass das Lebervenenblut kein Fibrin enthalte und nicht coagulire.

Es muss auffällig erscheinen, dass, obwohl diese Versuche zu einer Zeit ausgeführt worden sind, wo die Fibrinogenbestimmung noch gänzlich unvollkommen war, fast alle Physiologen bis in die neueste Zeit, zum grössten Theil ohne jegliche Controlle, sich dieser Ansicht angeschlossen haben.

Auch Dienst (26), welcher sich der Mathew'schen Anschauung von der Fibrinogenbestimmung anschliesst, sieht in der Leber ein für den Abbau dieses Eiweisskörpers hochwichtiges Organ und sagt, dass gerade in Folge der Leberschädigung bei der Eklampsie ein Circulus vitiosus schlimmster Art geschaffen werde, indem zu der vermehrten Bildung auch noch eine verringerte Zerstörung des Fibrinogens komme.

Allen diesen Theorien über die Entstehung bezw. Zerstörung des Fibrinogens ist durch die zahlreichen Arbeiten von Doyon, Gautier, Kareff, Morel und Nolf der Boden entzogen worden.

Diesen Autoren jst es gelungen, in einwandsfreier Weise den Nachweis zu führen, dass die Entfernung des Darmes entweder keinen Einfluss auf den Fibrinogengehalt hat oder sogar denselben erhöht, und dass auch nach der Darmentfernung einer Defibrination eine Wiederbildung des Fibrins folgt (37).

Auch die Lehmann'sche Behauptung von der Ungerinnbarkeit des Lebervenenblutes ist widerlegt, denn Nachprüfungen haben ergeben, dass dieses Blut immer gerinnt, zuweilen etwas schneller, zuweilen etwas langsamer als das Carotisblut (38).

Der Fibringebalt des arteriellen Blutes ist unter allen Umständen grösser als derjenige des entsprechenden Venenblutes. Nach Aderlass oder Totaldefibrination zeigt im Stadium der Fibrinregeneration das Lebervenenblut den grössten Fibringehalt (39).

Ferner konnten Doyon und Kareff (40) zeigen, dass, wenn nach Herausnahme der Leber die Circulation durch Verbindung der Pfortader mit einer Lebervene wieder hergestellt wird, das Fibrinogen verschwindet und das Blut ungerinnbar wird.

Nolf (41) bestätigt dieses und stellt ausserdem fest, dass das Lebervenenblut mehr Fibrinogen enthält als das Pfortaderblut. 
Landsberg, Untersucbungen über den Gehalt des Blutplasmas u.s.w. 709

\section{Die Leber als Fibrinogenbildner.}

Haben also die genannten Forscher in ihren Arbeiten einerseits die bis dahin ziemlich allgemein geltenden Ansehauungen von dem Fibrinogen in einwandsfreier Weise widerlegen können, so glauben sie andererseits in der Leber das Organ vor sich zu haben, welches die Bildungsstätte dieses Eiweisskörpers darstellt.

In einer grossen Reihe von Versuchen konnten sie den Nachweis erbringen, dass sowohl die Herausnahme der Leber, die Anlegung der Eck'schen Fistel, wie auch die Schädigung der Leberzellen durch die verschiedensten Gifte z. B. Chloroform, Phosphor, hepatoxisches Serum a. A. m. ein Verschwinden des Fibrinogens und ein Ungerinnbarwerden des Blutes nach sich zieht.

Zweifellos wirken alle diese hier nur sehr kurz angeführten Untersuchungen sehr überzeugend und lassen einen engen Zusammenhang zwischen Iseber und Fibrinogen bezw. Fibrin für sehr glaubhaft erscheinen.

\section{Das Knochenmark als Fibrinogenerzeuger.}

Im Gegensatz hierzu ist von deutscher Seite dem Knochenmark eine besondere Bedeutung für die Fibrinogenbildung beigelegt worden.

Von P. Th. Müller (42) ist der Nachweis geführt worden, dass nach künstlieher Infection von Thieren mit Typhusbacillen, Streptokokken und namentlich Eiterstaphylokokken ausser einer beträchtlichen Fibrinogenvermehrung im Blute eine solche sich auch im Knochenmark nachweisen lässt. Diese Erscheinung ist nicht durch den Blut- und Lymphgehalt dieses Organes zu erklären, sondern weist vielmehr darauf hin, dass dieser Eiweisskörper im Knochenmark selbst entsteht. Müller äussert die Ansicht, dass ausser dem Knochenmark auch den Lymphdrüsen, der Milz und auch dem reichen lymphoiden Apparat des Verdaungscanals die Fähigkeit der Fibrinogenerzeugung zukomme, während er den im Kreislauf befindlichen Leukocyten diese Kraft abspricht.

Diese Anschaung hat neuerdings eine starke Stütze erhalten durch die Arbeit von Morawitz und Rehn (43).

Diese Autoren zeigten, dass bei Thieren, die man im Stadium der Refibrination tödtet, neben einer starken Leukocytose eine erhebliche myeloide Reaction des Knochenmarkes und myeloide Herde in der Milz sich finden. 
710 Landsberg, Untersuchungen über den Gehalt des Blutplasmas u.s.w.

Verfährt man so, dass eine Defibrination des entnommenen und nachher wieder injicirten Blutes durch Hirudin verhindert wird, so bleiben diese Veränderungen aus.

Es werden also trotz der ausführlichen Arbeiten der oben genannten französischen Forscher immer wieder Untersuchungen zur Ermittelung der Ursprungsstätte des Fibrinogens angestellt, und dieses ist in verschiedenen Umständen begründet.

Die Schlussfolgerungen, welche Doyon und seine Mitarbeiter aus ihren Versuchen gezogen haben, werden nämlich etwas fraglich durch die Thatsache, dass das Blut von Thieren bei Phosphorvergiftung stark fibrinolytisch wirkt $(44,45,49)$, und die Vermuthung lässt sich nicht von der Hand weisen, dass es sich bei der Ungerinnbarkeit des Blutes nach Leberschädigung um einen beschleunigten Abbau des Fibrinogens bezw. Febrins handelt. Der Zusammenhang ist etwa so zu denken, dass die normale Leber ein gegen das fibrinolytische Ferment wirksames Antiferment producirt.

Hierzu möchte ich allerdings bemerken, das durch weitere Versuche erst die Frage entschieden werden muss, ob bei den vielen anderen Leberschädigungen, die nach Doyon zu einem Fibrinogenschwund führen, das Blut ebenfalls eine stark fibrinolytische Eigenschaft zeigt.

Bedeutungsvoll erscheint mir auch die Thatsache, dass nach Leberschädigung nicht immer das Ungerinnbarwerden des Blutes auf einer Fibrinogenabnahme beruht. Vielmehr findet sich z. B. nach Atropininjection in die Pfortader das erstere ohne das letztere (46).

Schliesslich findet bei der Anschauung jener französischen Autoren ein wesentliches Moment gar keine Berücksichtigung, nämlich das fast constant beobachtete Zusammentreffen von Hyperleukocytose mit Fibrinvermehrung. Dieser Umstand wird ja, wie schon erwähnt, auch von Mathew (34) für seine Theorie ins Feld geführt, und in dieser Hinsicht sind gerade die genannten Versuche von Müller (42), Morawitz und Rehn (43) sehr beachtenswerth.

Pfeiffer (47) führte als erster einwandsfrei den Nachweis, dass bei denjenigen Infectionskrankheiten, die mit einer starken Leukocytose einherzugehen pflegen, auch stets eine erhebliche Fibrinvermehrung zu beobachten ist, und er giebt folgende Mittelwerthe an: 
Landsberg, Untersuchungen über den Gehalt des Blutplasmas u.s.w. 711

Normal . . . . . $39,3 \mathrm{mg}$ N-Fibrin $=2,324$ pM. Fibrin

Pneumonie . . . $105,9 n n=6,26 n n$

Gesichtserysipel . . $89,0, n \quad n=5,26 \quad n \quad n$

Ac. Polyarthritis . . 127,5 $n "=7,54 " n n$

Scarlatina. . . . $85,7 n "=7,07 n n$

Lungenabscess . . $83,2 " n \quad=4,92 \quad n$ "

Puerp. Inf. mit para-

metr. Exsud. und diff.

Peritonitis . . . $\left.104,2 \quad n \quad n=6,16 \quad n \quad n^{1}\right)$

Hingegen zeigen Typhus, Malaria, Sepsis ohne locale Eiterung, Nephritis (Urämie) sowohl in Bezug auf Leukocytenzahl wie auch auf Fibrinmenge ein entgegengesetztes Verhalten.

Langstein und Meyer inficirten Kaninchen mit versehiedenen Mikroorganismen und kamen zu dem Ergebniss, dass neben den Streptokokken vor allem die Pneumokokken die Fähigkeit besitzen, eine starke Fibrinvermehrung hervorzurufen.

Die Versuche von Müller (42), welche zeigten, dass eine Infection mit Eiterstaphylokokken ganz im Gegensatz zu Sputumstaphylokokken von einer sehr erheblichen Steigerung der Fibrinogenmenge im Blut und im Knochenmark gefolgt ist, habe ich bereits Erwähnung gethan. Bekanntlich rufen diese Bakterien stets auch eine starke Leukocytose hervor.

In der Hallenser Frauenklinik ist die Beobachtung gemacht worden, dass bei Streptokokkeninfectionen das Blut ausserordentlich leicht gerinnt, und auch Much (48) sagt in einer neueren Arbeit, dass das Blut bei Streptokokken-, Staphylokokken-, Pneumokokken- und Coliinfection eine sehr starke Gerinnungsbeschleunigung erfährt, so dass es meist schon in der Spritze gerinnt.

Aus diesen Versuchen ergiebt sich also, dass bei allen Infectionskrankheiten mit stärkerer Hyperleukocytose auch eine beträchtliche Vermehrung der Blutgerirnungsfähigkeit und des entstehenden Fibrins zu beobachten ist.

Ein Vergleich lehrt, dass die hier vorhandenen Fibrinwerthe, die für die Eklampsie von Kollmann (13) gefundenen, ausserordentlich hohe Zahlen erreichen, während sie diejenigen von Dienst (14)

1) Eigene Berechnung. Der Stickstoffgehalt des Fibrins beträgt nach Hammersten $16,91 \mathrm{pCt}$. 
712 Landsberg, Untersuchungen über den Gehalt des Blutplasmas u.s.w.

an Höhe bedeutend übertreffen. Diese Thatsache möchte ich mit Nachdruck hervorheben.

Bei Infectionskrankheiten sind nach der Feststellung von Arneth (27) die ein- und zweikernigen Leukocyten stark in der Mehrheit, und auf diesen Unterschied gegenüber der Leukocytose bei der Schwangersehaftsniere und Eklampsie macht Dienst besonders aufmerksam.

Auf Grund seiner Anschauungen über die Herkunft der fibrinbildenden Substanzen muss er zu dem Schlusse gelangen, dass bei den Infectionskrankheiten diese Körper eine erheblichere Steigerung nicht erfahren. Dieses entspricht aber keineswegs den thatsächlichen Verhältnissen.

Kurz möchte ich noch darauf hinweisen, dass Pfeiffer (10) gezeigt hat, dass bei der Leukämie eine Vermehrung des Fibrinogens trotz der ausserordentlich hohen Leukocytenzahl nicht zu beobachten ist.

Im Gegensatz hierzu hat freilich neuerdings Erben (11) in einem von zwei untersuchten Fällen von myeloider Leukämie eine nicht unbeträchtliche Fibrinvermehrung auf 0,5944 pCt. herausgefunden, während er eine Fibrinsteigerung auch hier nicht beobachtet hat.

Vorläufig wird man wohl an der Ansicht festhalten müssen, dass das Fibrinogen bei der Leukämie keine Zunahme erfährt.

Es will mir scheinen, als ob in dem festgestellten Unterschiede zwischen der Leukocytose bei Leukämie und bei infectiösen - toxischen Erkrankungen nichts Befremdendes zu erblicken ist, worauf auch schon Müller in seiner Arbeit hingewiesen hat, denn es handelt sich doch hierbei um zwei ganz verschiedenartige Vorgänge.

Bei der Leukämie stellt die Leukocytose eine eigentliche Krankheitserscheinung dar, während man in der Leukocytose bei Infectionskrankheiten eine ausgezeichnete Vertheidigungsmaassregel des Organismus gegen den eingedrungenen Feind erblicken muss.

Bedenkt man dieses, so wird man unwillkürlich dem Gedanken zuneigen, dass es sich vielleicht bei der Vermehrung der fibrinbildenden Stoffe auch um eine Selbsthülfe des Körpers handelt.

Diese Ansicht wird besonders auch von Bergell (40) getheilt, auf dessen ausführliche Bearbeitung dieses Gegenstandes ich an dieser Stelle verweisen will. 
Landsberg, Untersuchungeu über den Gehalt des Blutplasmas u.s.w. 713

\section{Herkunft der Thrombokinase.}

Bereits Alex. Schmidt stellte den Satz auf, dass die zymoplastischen Substanzen bezw. das Fibrinferment aus den Leukocyten stammen und bei deren extravasculärem Zerfall frei werden.

Diese Anschauung war längere Zeit unbestritten und auch heute kann sie noch nicht als völlig widerlegt angesehen werden. Aber es mehren sich doch die Stimmen, welche sich zum Theil gegen einen Zerfall, zum Theil gegen jegliche Betheiligung der weissen Blutkörperchen bei der Fibrinfermentbildung wenden.

Es lässt sich heute mit ziemlicher Bestimmtheit sagen, dass ein starker. Leukocytenzerfall zur Fibrinfermentbildung nicht erforderlich ist.

Rüchel und Spitta (50) und Bayon (51) haben gezeigt, dass die Leukocytenabnahme bei der Gerinnung im Einzelnen eine sehr wechselnde Höhe erreicht, im Durchschnitt sich aber in recht bescheidenen Grenzen hält. Man kann der Ansicht dieser Autoren wohl zustimmen, dass dieser geringe Leukocytenverlust sich durch den sehr wechselnden Einschluss von weissen Blutkörperchen in das Fibrinnetz erklären lässt.

Rüchel und Spitta weisen auch namentlich darauf hin, dass eine besondere Abnahme der polynucleären Leukocyten nicht zu beobachten ist, sondern dass vor und nach der Gerinnung das relative Verhältniss der einzelnen Leukocytenklassen keine Verschiebung aufweist.

Krüger (52) hat sich mit grosser Schärfe gegen diese Feststellungen gewendet. Aber abgesehen von dem Umstande, dass die Auslegung, welche er den Resultaten der genannten Autoren giebt, kaum richtig sein dürfte, übersieht er die zahlreichen anderen, besonders von französischer Seite über diesen Gegenstand veröffentlichten Untersuchungen, welche alle gegen die von Krüger vertheidigte Ansicht sprechen.

Dastre (53) und Arthus (54) führen in ihren Arbeiten aus, dass die Fibrinfermententstehung weder an einen Zerfall noch an eine Degeneration der weissen Blutkörperchen geknüpft ist, und sie glauben, dass es sich hier um einen secretorischen Vorgang handelt.

Gegen eine Betheiligung der polynucleären Leukocyten bei der Blutgerinnung wendet sich neben Dastre besonders auch Stassano, 
714 Landsberg, Untersuchungen über den Gehalt des Blutplasmas u.s.w.

welcher die Bedeutung der Mononucleären ganz in den Vordergrund schiebt.

Im Gegensatz zu diesen französischen Forschern, die eine Bedeutung der Leukocyten für die Blutgerinnung keineswegs leugnen, sprechen sich andere namhafte Autoren, ich nenne nur Bürker, mit Entschiedenheit gegen jegliche Betheiligung der Leukocyten aus und sind der Ansicht, dass nur die Blutplättchen bei der Gerinnung eine Rolle spielen.

Es kann als erwiesen gelten, dass der Blutgerinnung bezw. Thrombosenbildung eine ausgiebige Agglutination von Blutplättchen vorausgeht, und dass bei der Gerinnung selber dieselben massenhaft zerfallen.

Bürker (56) stellt auf Grund seiner Untersuchungen eine directe Beziehung zwischen Beeinflussung von Blutplättchen und Blutgerinnung fest, während er eine Aenderung im Zustande der anderen Blutelemente für völlig irrelevant hält.

Gegen diese Auffassung ist von verschiedenen Seiten geltend gemacht worden, dass Flüssigkeiten, welche keine Blutplättchen enthalten, wie Gansblut, Lymphe und von Blutplättchen befreites Säugethierblut spontan, allerdings sehr langsam gerinnen.

Dieser Einwand erscheint mir wenig zwingend. Die Leukocyten und ebenso auch die rotben Blutkörperchen enthalten, wie überhaupt jede Zelle, thrombokinatisch wirkende Substanzen. Eine nur leukocytenhaltige Flüssigkeit muss daher, vorausgesetzt, dass alle sonstigen zur Fibrinbildung nöthigen Substanzen in ihr vorhanden sind, auch der Gerinnung anheimfallen. Dieser Vorgang wird jedenfalls durch Zusatz von Thrombokinase stark beschleunigt.

Manches wird noch für, Manches gegen die Betheiligung der Leukocyten bei der Fibrinfermentbildung angeführt. Man kann beute noch kein abschliessendes Urtheil darüber geben, ob die von Bürker aufgestellte Theorie sich wird im ganzen Umfange aufrecht erhalten lassen. Jedenfalls spricht Vieles dafür. Ziemlich allgemein vertritt man jetzt bereits den Standpunkt, dass den Blutplättchen zum Mindesten die wichtigste Rolle bei dem Gerinnungsvorgange zukommt, und die alte Schmidt'sche Lehre verliert mehr und mehr an Boden.

Ich habe bereits soeben der schon lange bekannten, besonders von Rauschenbach (57) festgestellten Thatsache Erwähnung gethan, dass alle Zellen gerinnungserregende bezw. -befördernde Stoffe 
Landsberg, Untersuchungen über den Gehalt des Blutplasmas u.s.w. 715 enthalten, und dass die Thrombokinase ein ganz allgemeines Protoplasmaprodukt ist.

Sehr interessant erscheint mir in dieser Hinsicht die von Much (48) in seiner oben erwähnten Arbeit gemachte Mittheilung, dass bei Einsaat von Staphylococeus aureus in menschliches Blutplasma nach kurzer Zeit eine Gerinnung eintritt. Much ist geneigt, in diesem Mikroorganismus eine der Thrombokinase gleichwirkende Staphylokinase anzunehmen.

lch erachte es für wichtig, auf diesen Punkt der allgemeinen Verbreitung thrombokinatischer Substanzen einzugehen.

Einzelne Autoren sehen neuerdings in dem Uebergang von Fibrinferment aus der Placenta in das mütterliche Blut ein wesentliches ätiologisehes Moment für das Zustandekommen der Eklampsie.

Diese Anschauung stützt sich namentlich auf die festgestellte Thatsache, dass Placentarpresssaft bei Thieren, intravenös injicirt, tödtlich wirkt, und dass die Section in diesen Fällen eine sehr ausgedehnte Thrombosenbildung ergicbt.

Diese Arbeiten stellen nur eine Ergänzung zu der längst bekannten Thatsache dar, dass Gewebsextrachte, intravenös injicirt, stark gerinnungsanregend wirken.

In neuerer Zeit haben sich besonders Conradi (58), Boggs (59) und Mioni (60) mit diesem Gegenstande beschäftigt. Sie haben fast alle Organe nach dieser Hinsicht ausführlich untersucht und sind zu dem Ergebniss gelangt, dass namentlich den kernreichen Organen wie Pankreas, Thymus, Lymphdrüsen eine stark gerinnungserregende Kraft innewohnt, welehe aber auch Lober, Niere, Milz u.s. w. in mehr oder weniger ausgedehntem Maasse besitzen, was auch von Freund (61) bestätigt wird.

Nach alle dem kann es nur natürlich erscheinen, dass auch der Salt aus der Placenta mit ihrem grossen Kernreichthum bei intravenöser Injection eine starke Thrombosirung hervorruft.

Ich beabsichtige nicht, an dieser Stelle auf die sehr verschiedenen Ansichten einzugehen, welche über die Natur der in den Gewebssäften vorhandenen gerinnungserregenden Substanzen besteben. Die Erforsehung dieser ebenso interessanten wie schwierigen Frage ist noch weit entfernt von einem einheitlichen Resultat.

Hinweisen möchte ich aber auf den Unterschied, welcher in der Wirkung intravenöser Injection von Gewebssaft und Fibrinferment $\mathrm{zu}$ constatiren ist.

Bereits Naunyn (62) und Jakowicky (63) konnten darthun, 
716 Landsberg, Untersuchungen über den Gehalt des Blatplasmas u.s.w.

dass Injectionen von Fibrinferment keine intravasculären Gerinnungen hervorrufen. Diese älteren Ergebnisse sind neuerdings von Mioni (60) und Boggs (59) vollauf bestätigt worden. Letzterer zeigte, dass $40 \mathrm{ccm}$ von activirtem Serum, welches auf Fibrinogenlösung stark wirkte, beim Kaninchen intravenös injicirt gar keinen Einfluss auf die Gerinnung ausübt.

Hiernach muss es zum Mindesten sehr fraglich erscheinen, ob es berechtigt ist, die Gewebssaftwirkung mit der Wirkung von Fibrinferment bezw. deren Vorstufe zu identificiren. Vielmehr scheint es sich hier um sehr complicirte Verhältnisse zu handeln. Die Anwesenheit von Fibrinogen und Fibrinferment im Blute ist noch keineswegs immer gleichbedeutend mit einer eintretenden Gerinnung, sondern es spielen dabei die verschiedenartigen gerinnungsauslösenden bezw. gerinnungsverhindernden Momente eine bedeutungsvolle Rolle.

Unsere Kenntnis aller dieser Dinge ist noch sehr gering. und daher ist äusserste Vorsicht bei ihrer Bewerthung erforderlich, zumal wenn es sich um pathologische Zustände handelt.

\section{Zusammenfassung.}

Zum Schluss möchte ich die wichtigsten Ergebnisse meiner Untersuchungen und meiner theoretischen Betrachtungen kurz zusammenstellen.

Der Cresammteiweissgehalt ist bei Schwangeren und Kreissenden etwas geringer als bei Nichtschwangeren, bei den Neugeborenen niedriger als bei den Müttern.

Der Fibringehalt ist bei Schwangeren etwas höher als bei nichtschwangeren Frauen, bei Kreissenden noch höher als bei Schwangeren, bei Neugeborenen selbst niedriger als bei Nichtschwangeren.

Eine Steigerung der Fibrinogenmenge bei Eklamptischen gegenüber normalen Kreissenden ist nicht nachweisbar.

Bei dem untersuchten Fall von Schwangerschaftsniere ist der Fibrinogengehalt nicht vermebrt. Hingegen zeigt die Reststickstoffmenge eine sehr starke Erhöhung.

Die im Kreislauf befindlichen Leukocyten haben mit der Fibrinogenbildung nichts zu thun. Als Entstehungsort für das Fibrinogen kommen in Betracht einerseits die Leber, andererseits die lymphoiden Organe, und zwar besonders das Knochenmark.

Eine stärkere Betheiligung der Leukocyten bei der Fibrinfermentbildung ist äusserst zweifelhaft. Jedenfalls kann die Be- 
Landsberg, Untersuchungen über den Gehalt des Blutplasmas u.s.w. 717

hauptung als widerlegt angesehen werden, dass zur Fibrinfermentbildung ein stärkerer Zerfall von Leukocyten, vornehmlich der polynucleären, erforderlich ist.

Im Gegensatz hierzu gewinnt die Ansicht, dass die Blutplättchen für die Gerinnung von grösster Bedeutung sind, immer mehr an Boden.

Gegen eine vornehmliche Betheiligung der vielkernigen polynucleären Leukocyten sprechen die Verhältnisse bei den Infectionskrankheiten. Obwohl diese Blutelemente hier in starker Minderheit sind, hat sich eine sehr beträchtliche Steigerung der Blutgerinnungsfähigkeit und der entstehenden Fibrinmenge herausgestellt.

Nach Allem komme ich zu dem Ergebniss, dass die Leukocyten als ätiologisches Moment für die Schwangerschaftsniere und Eklampsie im Sinne von Dienst nicht in Betracht kommen.

Auch glaube ich nicht, dass es berechtigt ist, aus der thrombosenerzeugenden Wirkung von Placentarsaftinjection den Schluss zu ziehen, dass das Fibrinferment den wesentlichen Factor bei der Entstehung der Eklampsie darstellt.

\section{Li t e ratur.}

1. J. Veit, Untersuchungen über den osmotischen Druck zwischen Mutter und Kind. Zeitschr. f. Geburtshülfe. 1900. Bd. 42. S. 316.

2. Heymann, Folia haematologica. 1906. Bd. 3. S. 7 u. 71.

3. Zangemeister, Die Beschaffenheit des Blutes in der Schwangerschaft u. d. Geburt. Zeitschr. f. Geburtshülfe. 1903. Bd. 49. S. 92.

4. Zangemeister und Meissl, Vergl. Untersuchungen ïber mütterl. und kindl. Blut. Münch. med. Wochenschr. 1903. S. 673.

5. Lewinski, Beobachtungen über den Gehalt des Blutplasmas an SerumAlbumin-Globulin und Fibrinogen. Pflüger's Arch. 1903. Bd. 100. S. 601.

6. Scipiades und Farkas, Ueber die moleculare Concentration des Blutserums. Hegar's Beitr. 1905. Bd. 9. S. 84.

7. Raineri, Archivo di Ostetricea e Gynecologia Napoli 1903. Im Ref. Fol. haemat. 1907. Bd. 4. S. 213.

8. Bauereisen, Die Beziehungen zwischen dem Eiweiss der Frauenmilch u. d. Serumeiweiss von Mutter u. Kind. Dieses Arch. 1910. Bd. 90 . S. 349.

9. Langstein und Meyer, Ueber das Verhalten der Eiweisskörper d. Blutplasmas bei exper. Infection. Hofmeister's Beitr. 1904. Bd. 5. S. 69.

10. Pfeiffer, Ueber den Fibringehalt d. leukäm. Blutes. Centralbl. f. innere Med. 1904. S. 809.

11. Erben, Die chem. Zusammensetzung d. leuk. Blutes. Zeitschr. für klin. Med. 1908. Bd. 66. S. 278. 
718 Landsberg, Untersuchungen über den Gehalt des Blutplasmas u.s.w.

12. Nasse, Das Blut von Schwangeren. Dieses Arch. 1876. Bd. 10. S. 315.

13. Kollmann, Zur Aetiologie n. Therapie d. Ekiampsie. Centralbl. f. Gyn. 1897. Bd. 2. S. 341 .

14. Dienst, Krit. Studien über d. Pathogenese d. Eklampsie. Dieses Arch. 1902. Bd. 65 . S. 369.

15. Arthus et Pagès, Nouvelle théorie chimique de la coagulation du sang. Arch. de Phys. 1890. 5. Série. 2. Bd. S. 739.

16. Hammersten, Ueber d. Bedeutung d. lösl. Kalksalze für d. Faserstoffgerinnung. Zeitschr, f. physiol. Chemie. 1896/97. Bd. 22. S. 333.

17. Reye, Ueber Nachweis und Bestimmung des Fibrinogens. Inaug.-Diss. Strassburg 1898.

18. Denis, Nouv. études chim. physiol. et médic. sur les substances albuminoides. Paris 1856. (Cit, in Virchow's Jahresbericht.)

19. Heubner, Die Spaltung des Fibrinogens bei der Fibringerinnung. Arch. f. Path. u. Pharm. 1903. Bd. 49. S. 229.

20. Hu iscamp, Zur Globulinfrage. Zeitschr. f. physiol. Chem, 1905. Bd.44. S. 182.

21. Kauder, Zur Kenntniss der Eiweisskörper des Blutserums. Arch. f. Path. und Pharm. 1886. Bd. 20. S. 411.

22. Hammersten, Lehrb. d. physiol. Chemie. 1910.

23. Hoppe-Seyler-Thierfelder, Handb. d. physiolog. u. patholog. chem. Analyse. 1909. a) S. $560-62$, b) S. 393.

24. Schmor1, 1) Pathol.-anat. Unters. über Eklampsie. Leipzig 1893. 2) Zur pathol. Anat. d. Eklampsie. Verh. in d. Ges. f. Gyn. Giessen 1901.

25. Lubarsch, Ueber d. pathol. Anat. u. Pathogenese d. Eklampsie. Corresp.Bl. d. allg. meckl. Aerztevereins.

26. Djenst, Die ätiol. Bedeut. d. weissen Blutkörp. f. d. Schwangerschaftsnierè u. Eklampsie. Dieses Arch. 1910. Bd. 90. S. 536.

27. Arneth, J) Die neutrophilen Leukocyten etc. Jena 1904. Vergl. Hiller, Folia Haem. Bd, 2. S. 85. - 2) Die Leukocyten in der Schwangersch. etc. Dieses Arch. Bd. 74. S. 143.

28. Alex. Schmidt, 1) Die Lehre von den fermentativen Gerinnungserscheinungen.Dorpat 1876. - 2) Zur Blutlehre. Leipzig 1892. (Beides cit. nach Virch. Jahresber.)

29. Hammersten, Ueber d. Faserstoff u. seine Entstehung aus d. Fibrinogen. Pflüger's Arch. 1883. Bd. 30. S. 437.

30. Norawitz, 1) Beitr. z. Kenntn. d. Blutgerinnung. Deutsch. Arch. f. klin. Med. 1904. Bd. 79. S. 1. - 2) Chem. d. Blutgerinnung. Erg. d. Phys. 1905. Bd. 4. S. 307. - 3) Die Blutgerinnung. Handb. der Biochemie von Oppenheimer. 1909. Bd. II. 2. S. 52.

31. Fuld und Spiro, Der Einfluss gerinnungshemmender Agentien. Hofmeister's Beitr. 1904. Bd. 5. S. 171.

32. Joh. Müller, Beob. z. Analyse d. Lymphe, d. Blutes u. d. Chylus. Poggendorf's Annalen, Bd. 25. 1832. S. 537.

33. Dastre, Évolution du fibrinogène dans le sang. Arch. de Phys, 1894. Bd. 25. (Hier auch ältere Literatur.)

34. Mathew, The origine of Fibrinogen. Americ. Journ. of Phys. 1899. Bd. 3. S. 53. 
35. Corin und Ansiaux, Unters. äb. Phosphorvergiftung. Vierteljahrsschr. für gericht. Med. 1894. 3. Foige. Bd. 7. (Cit. nach. Virch. Jahresber.)

36. Lehmann, Jahresb. für practische Chemie. 1851. (Cit. nach Cannstadt's Jahresb. 1855.)

37. Doyon et Gautier, Sur le rôle de l'intestin dans la fibrinogenèse. Journ. de Phys. et de Pathol. génér. 1907. Bd. 90. S. 405.

38. Doyon, Gautier et Kareff, Recherches sur la coagulabilité du sang des veines sus-hépatiques. Ebendaselbst. 1906. Bd. 8. S. 1003.

39. Doyon, Morel et Kareff, Teneur comparée du sang en fibrine dans différentes territoires vasculaires. Ebendaselbst. S. 783.

40. Doyon et Kareff, Modifications de la coagulabilité du sang consécutives a la déstruction du foie. Ebendaselbst. 1905. Bd. 7. S. 639.

41. Nolf, Les modifications de la coagulation du sang chez le chien après l'exstirpation du foie. Arch. int. d. Phys. 1905. Bd. 3.

42. P. Th. Müller, Ueber chem. Veränd. d. Knochenmarks nach intraperitonealer Bacterieneinspritzung. Hofmeister's Beitr. 1905. Bd: 6. S. 454, u. Phys. Centralbl. 1906.

45. Morawitz u. Rehn, Zur Kenntniss d. Entstehung d. Fibrinogens. Arch. f. Pathol, u. Pharm, 1908. Bd. 58. S. 141.

44. Jakoby, Ueber d. Bezieh. d. Leber- u. Blutveränderungen zur Autolyse. Zeitschrift f. physiol. Chemie, 1900. Bd. 30. S. 174.

45. Loeb, Einige neuere Arb. über d. Blutgerinnung bei Wirbellosen und Wirbelthieren. Bioch. Centralbl. 1907. Bd. 6. S. 829-884.

46. Doyon et Kareff, Action de l'atropine sur la coagulation du sang. Journ. d. Phys. et de Pathol. gén. 1906. Bd. 18.

47. Pfeiffer, Ueber den Fibringehalt d. menschl. Blutes. Zeitschr. f. klin. Med. 1897. Bd. 33. S. 215.

49. Much, Ueber eine Vorstufe d. Fibrinfermentes in Culturen von Staphylococcus aureus. Bioch. Zeitschr. 1908. Bd. 14. S. 143.

49. Bergell, Ueber die Wirkung des Fibrins. Deutsche med. Wochenschrift. 1908. No. 9 u. 1909. S. 663.

50. Rüchel u. Spitta, Einige Beob. über Blutgerinnung and Leukocyten. Arok, f. Pathol. u. Pharm. 1904. Bd. 49. S. 285.

51. Bayon, Leukocyten und Blutgerinnung. Bioch. Zeitschr. 1904. Bส. 45. S. 104.

52. Krüger, Leukocyten uad Blutgerinnung. Arch. f. Pathol. u. Pharmak. 1904. Bd. 51. S. 325.

53. Dastre, Sur les causes initiales de la coagulation etc. Compt. rend, de la Soc. de Biol. 1903. Bd. 55.

54. Arthus, Sur la génèse du fibrinferment. Ebendort. Beides cit. n. Journ. de Phys. et de Path. gén. 1904. Bd. 6.

55. Stassano, Rôle des diverses espèces de leucocytos dans la coagulation du sang. Ebendaselbst.

56. Bürker, Blutplättchen und Blutgerinnung. Pflüger's Archiv. 1903/04. Bd. 102. S. 36 .

57. Rauschenbach, Ueber die Wochselwirkungen zwischen Protoplasma und Blutplasma. In.-Diss. Dorpat 1883. 
720 Landsberg, Untersuchungen über den Gehalt des Blutplasmas u.s.w.

58. Conradi, Ueber die Beziehungen der Autolyse zur Blutgerinnung. Hofmeister's Beitr. 1902/03. Bd. 1. S. 136.

59. Boggs, Ueber Beeinflussung der Gerinnungszeit im lebenden Organismus. Dentsches Arch. f. klin. Med, 1904. Bd. 79. S. 539.

60. Mioni, Contributions à l'étude des transf. sang. Arch. int. de Phys. 1904/05. Bd. 3.

61. Freund, Zur placentar. Eklampsie-Aetiologie. Berliner klin. Wochenschr. 1909. No. 15.

62. Naunyn, Untersuchungen über Blutgerinnung im lebenden Thiere. Arch. f. Pathol. u. Pharm. 1873, Bd. 1, S. 1.

63. Jakowicky, Zur physiologischen Wirkung der Bluttransfusion. In.-Diss, Dorpat 1875 . 